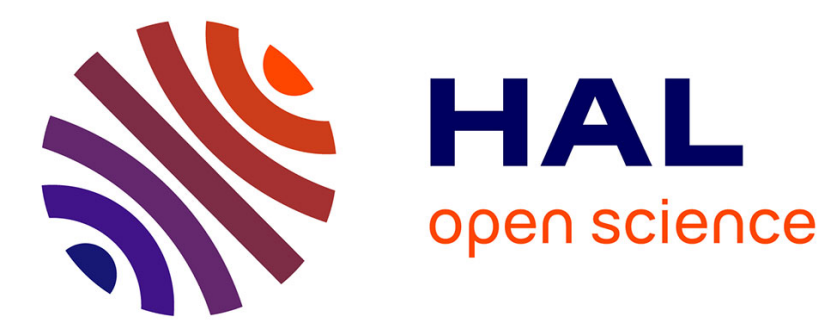

\title{
Color selectivity lent to a cholesteric liquid crystal by monitoring interface-induced deformations
}

Gonzague Agez, Rajaa Bitar, Michel Mitov

\section{To cite this version:}

Gonzague Agez, Rajaa Bitar, Michel Mitov. Color selectivity lent to a cholesteric liquid crystal by monitoring interface-induced deformations. Soft Matter, 2011, 7 (6), pp.2841-2847. 10.1039/C0SM00950D . hal-01730536

\section{HAL Id: hal-01730536 \\ https://hal.science/hal-01730536}

Submitted on 24 Feb 2022

HAL is a multi-disciplinary open access archive for the deposit and dissemination of scientific research documents, whether they are published or not. The documents may come from teaching and research institutions in France or abroad, or from public or private research centers.
L'archive ouverte pluridisciplinaire HAL, est destinée au dépôt et à la diffusion de documents scientifiques de niveau recherche, publiés ou non, émanant des établissements d'enseignement et de recherche français ou étrangers, des laboratoires publics ou privés. 


\section{Color selectivity lent to a cholesteric liquid crystal by monitoring interface-induced deformations ${ }^{\dagger}$}

\section{Gonzague Agez, Rajaa Bitar, and Michel Mitov}

The cholesteric liquid crystalline structure is omnipresent in living matter and concerns many applications in optics because of its property of selective light reflection. The color reflected by this material depends on material parameters such as the molecular chirality or the concentration of chiral dopant, the helical pitch of the twisted structure and the optical indices. In the present publication, we show that the color may be selected simply by varying the annealing time of a cholesteric oligomer film with hybrid anchoring. Experimental and simulated transmittance spectra reveal the color shift. The three-dimensional representation of the structure is provided from the macroscopic scale to the mesoscale by combining complementary imaging techniques (optical microscopy, transmission and scanning electron microscopies, atomic force microscopy) to image the texture in different directions. We show that the color selectivity is due to controlled changes of the orientation of the helix axis with respect to the air-material interface. The optical behavior is described with a single parameter, which is the angle between the film surface normal and the orientation within the film of the helical axis. Potential applications in the field of chiro-optical devices concern self-assembled chiral microreflectors fabricated via a low-cost controllable thermal process. Additionally, the texture formation provides insight to aid the understanding of the exoskeleton morphogenesis of beetles like Chrysina Gloriosa.

Article history: Received $8^{\text {th }}$ Sep $2010 /$ Accepted 15 $5^{\text {th }}$ Dec $2010 /$ First published (online) $31^{\text {th }}$ Jan 2011.

https://doi.org/10.1039/C0SM00950D

Centre d'Elaboration de Matériaux et d'Etudes Structurales (CEMES), CNRS, Univ. Toulouse, 29 rue J. Marvig, 31055

Toulouse cedex 4, France.E-mail: mitov@cemes.fr; Fax : +33 562257999 ; Tel : +33 562257861

${ }^{\dagger}$ Electronic supplementary information (ESI) available : Supplementary data on optical and structural properties, Online movie. 


\section{Introduction}

Cholesteric liquid crystalline states of living matter are related to the organization of cholesterol esters [1], chromatin in vivo and in vitro [2-4], polysaccharides and cellulose derivatives [5, 6], collagen in cornea [7], bone [8], or fish scale [9], chitin in cuticles of crabs [10], insects [11], and many arthropods [12]. Applications [13] like supertwisted nematic liquid crystal displays, temperature and pressure sensors, tunable bandpass filters, polarizerfree reflective displays or smart window prototypes take advantage of the optical properties of the cholesteric phase.

A cholesteric liquid crystal (CLC) film may selectively reflect light because of its helical structure, which is associated with its molecular chirality. The mean reflection wavelength $\lambda_{0}$ is directly related to the helical pitch $p$ (structural periodicity that represents a rotation of $360^{\circ}$ in the orientation of the rod-like molecules) by Bragg's law: $\lambda_{0}=n p \cos \theta$, where $n$ is the average refractive index and $\theta$ is the angle between the direction of light propagation and the helix axis [14-16]. Circularly polarized light with the same handedness as the structure is reflected. In the absence of external control [17], the color selectivity therefore depends on the following material parameters: the molecular chirality or the concentration of chiral dopant, the pitch, and the optical indices.

In this report, we put forward a novel scenario to tune the reflection color of CLCs by simply tuning the annealing time of an open oligomer film that has hybrid anchoring. Transmittance spectra and investigations of structure using complementary imaging techniques reveal that the color versatility is due to controlled changes of the helix axis orientation with respect to the air-material interface. We shall also discuss applications in the field of chiro-optical devices and an analogy with the exoskeleton formation of some beetles. 


\section{Materials and methods}

\section{Materials}

CLC oligomers from Wacker Chemie $\mathrm{GmbH}$ were used. The molecule is a siloxane cyclic chain with two types of side chains attached via spacers: an achiral mesogen and a (chiral) cholesterol-bearing mesogen [18]. When coated on a glass or plastic substrate, the compound exhibits typical iridescent colors that range from blue to red upon tuning the molar percentage of chiral mesogens in the oligomer molecule; a percentage that can vary from $31 \%$ to $50 \%$ $[19,20]$. We have blended as-called Silicon Blue (SB) and Silicon Red (SR) compounds at 40:60 wt.\% to tune the mean position of the reflection band to the middle of the visible spectrum (i.e., $560 \mathrm{~nm}$ ). The cholesteric phase appears between $180-210{ }^{\circ} \mathrm{C}$ (clearing temperature range) and $40-50{ }^{\circ} \mathrm{C}$ (glass-transition temperature range). A thin film with cholesteric organization and the related light-reflection properties can easily be quenched below the glass-transition temperature. In this case it becomes a solid film that can be handled at room temperature.

\section{Film preparation}

Oligomer films $13 \mu \mathrm{m}$ thick are confined between glass plates separated by Mylar spacers. No peculiar surface treatment or alignment layers were used. The samples were kept at $140{ }^{\circ} \mathrm{C}$ for 2 minutes. Each sample was then quenched by swiftly putting it on a metallic substrate at room temperature. The cholesteric film changes from a viscous state to a glassy solid state. The sample was then kept at low temperature $\left(2{ }^{\circ} \mathrm{C}\right)$ to facilitate the clean removal of the cover glass and to obtain a smooth interface. This process yields an exposed film ready to be annealed at $140^{\circ} \mathrm{C}$. Between acquisitions of the transmittance spectrum, the sample was kept again at $140{ }^{\circ} \mathrm{C}$ to continue the annealing process until the next quenching. The preferential 
molecular orientation is planar on the glass substrate and homeotropic (perpendicular) at the air interface.

\section{Optical textures}

The optical texture of the films was investigated in transmission mode by using a polarizing microscope (AX70 from Olympus). The images were obtained by using a TRI CCD F22GV camera from Hitachi, which has a numerical resolution of $0.13 \mu \mathrm{m} /$ pixel.

\section{Transmittance properties}

The film transmittance was investigated at room temperature and at normal incidence by using a Cary 5000 spectrometer from Varian. The incident light was unpolarized.

\section{Preparation of samples for transmission electron microscopy}

A small piece of oligomer film was embedded in an epoxy resin that was then cured at $40{ }^{\circ} \mathrm{C}$ (below the glass transition temperature of oligomers). Thin slices were obtained with an ultramicrotome (UltraCut S from Reichert) by using a diamond knife at ambient temperature. The material was cut perpendicularly to the film surface (cross-sections) and 80-nm-thick slices were directly retrieved on carbon-coated grids.

\section{Transmission electron microscopy conditions}

The observations were performed using a transmission electron microscope (TEM, Philips CM30) operating at $300 \mathrm{kV}$ under normal conditions [21-23]. This means that the dose of electrons received by the specimen is higher than the critical dose which deletes the diffraction contrast, so the images are produced by thickness diffusion contrast subsequent to irradiation. The contrast was enhanced by means of a slight defocusing. 


\section{Scanning electron microscopy imaging}

We used a JSM-6490 scanning electron microscope (SEM) from JEOL. Samples were coated with a platinum-iridium alloy film to create a conductive surface and fixed on the support with carbon-coated adhesive. Investigations were performed using the secondary electron imaging mode.

\section{Atomic force microscopy imaging}

Atomic force microscopy (AFM) measurements were performed at room temperature and under ambient conditions using a Veeco Dimension 3100 Scanning Probe Microscope controlled by a NanoScope 3D controller. The images were obtained in tapping mode.

\section{Numerical simulations}

Simulations were performed using the finite-difference time-domain (FDTD) method [24] of the Meep software package [25] with subpixel smoothing for increased accuracy. The simulations were based on information provided from TEM cross-sectional images.

\section{Image analysis}

For each annealing time, cross-sections $13 \times 120 \mu \mathrm{m}^{2}$ in size were rebuilt from about ten TEM images. The resolution ranges from 16 to $25 \mathrm{~nm}$ per pixel. Because the twist axis is perpendicular to the lines in fingerprint texture (fig. 2), the t-vector (unitary vector along the helix axis) is detected by two-dimensional spatial Fourier Transform (FT) performed on windows $64 \times 64$ pixels in size (we used the free FFTW C subroutine library). The wave vector $q$ of the contrast modulation provides quasilocal information on the natural pitch value $p_{0}$ and the angle $\theta$. To scan the entire image, this FT analysis is carried out every 5 pixels in $x$ and $z$, each time sliding the $64 \times 64$ window. 


\section{Results and discussion}

\section{Optical properties from macroscale to mesoscale}

Figure 1a shows the reflection color range for normal incidence of samples deposited on a black substrate.
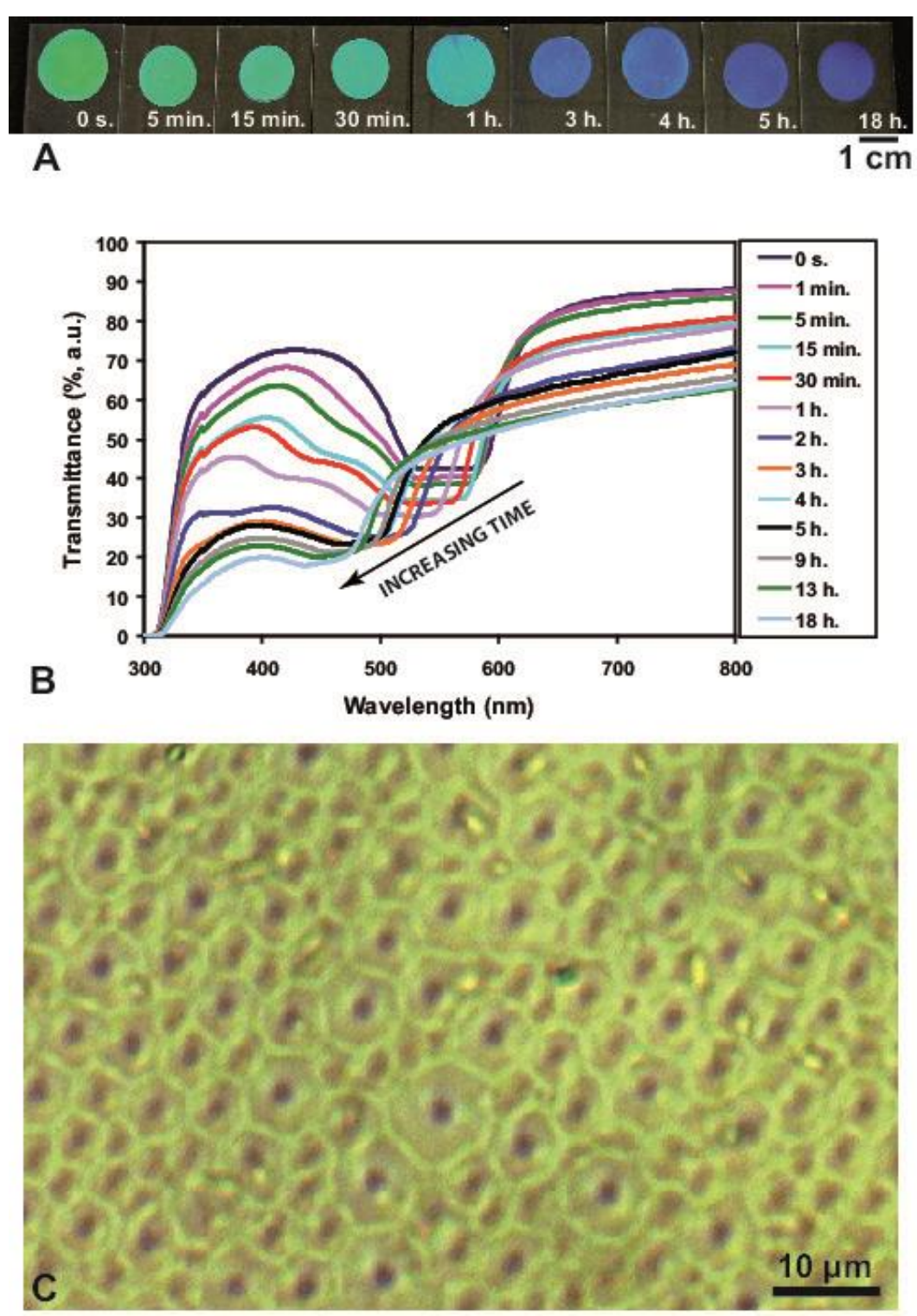

Fig. 1. Optical properties of cholesteric liquid crystalline films quenched at $140{ }^{\circ} \mathrm{C}$ for different annealing times (unpolarized light, room temperature). (A) Macroscopic samples deposited on a black substrate and observed at normal incidence. (B) Transmittance spectra for different annealing times. The position of the band gap progressively shifts towards smaller wavelengths as the annealing time increases. (The sudden decrease of light intensity below $400 \mathrm{~nm}$ corresponds to the cutoff wavelength 
of the glass substrate.) (C) An optical micrograph of the free surface shows an array of adjacent cells, which is the polygonal texture (transmission mode, annealing time $=3 \mathrm{~h}$, numerical aperture $=0.50$ ).

The reflection color changes gradually from green to blue as the annealing time increases from 0 to 18 hours. We investigated the transmittance properties upon quenching after different annealing times (fig. 1b). The mean position of the band gap shifts progressively from about $560(0 \mathrm{~h})$ to $460 \mathrm{~nm}(18 \mathrm{~h})$. This blue shift occurs without significant bandwidth broadening (fig. S1 of ESI†). When observed with unpolarized light using an optical microscope, the samples exhibit a mosaic of cells and colors as soon as the annealing time exceeds 1 minute (fig. 1c); this is referred to as the polygonal texture [26, 27]. Each cell appears like a dark greenish polygon with a central black spot and is surrounded by a bright green region.

\section{Structural investigations}

The fact that the quenched materials are in the solid state provides a unique opportunity to image the cross-sectional structure by TEM. The local distribution of the cholesteric periodicity as well as the orientation of the helical axis may be visualized from one interface to the other. Without annealing (fig. 2a), the planar organization permeates the entire layer and the cross-sectional image shows a network of periodic dark and bright lines parallel to both interfaces with some disclination defects; this pattern is called the fingerprint texture. The helical axis is everywhere perpendicular to the lines. For a cut in a direction normal to the axis, the distance between identical lines is approximately the half pitch (the cholesteric structure looks the same physically and has the same dielectric properties after a $180^{\circ}$ rotation). Inside the dark (bright) lines, the rod-like molecules are parallel (perpendicular) to the image plane [21]. Although fingerprint patterns in cholesteric textures have been described by optical microscopy many times since the observations of pioneers in the field of 
LC science [28], TEM imaging of these patterns in thermotropic cholesteric materials [29] and discussions of the origin of the related line contrast are rarer [21-23, 30, 31].
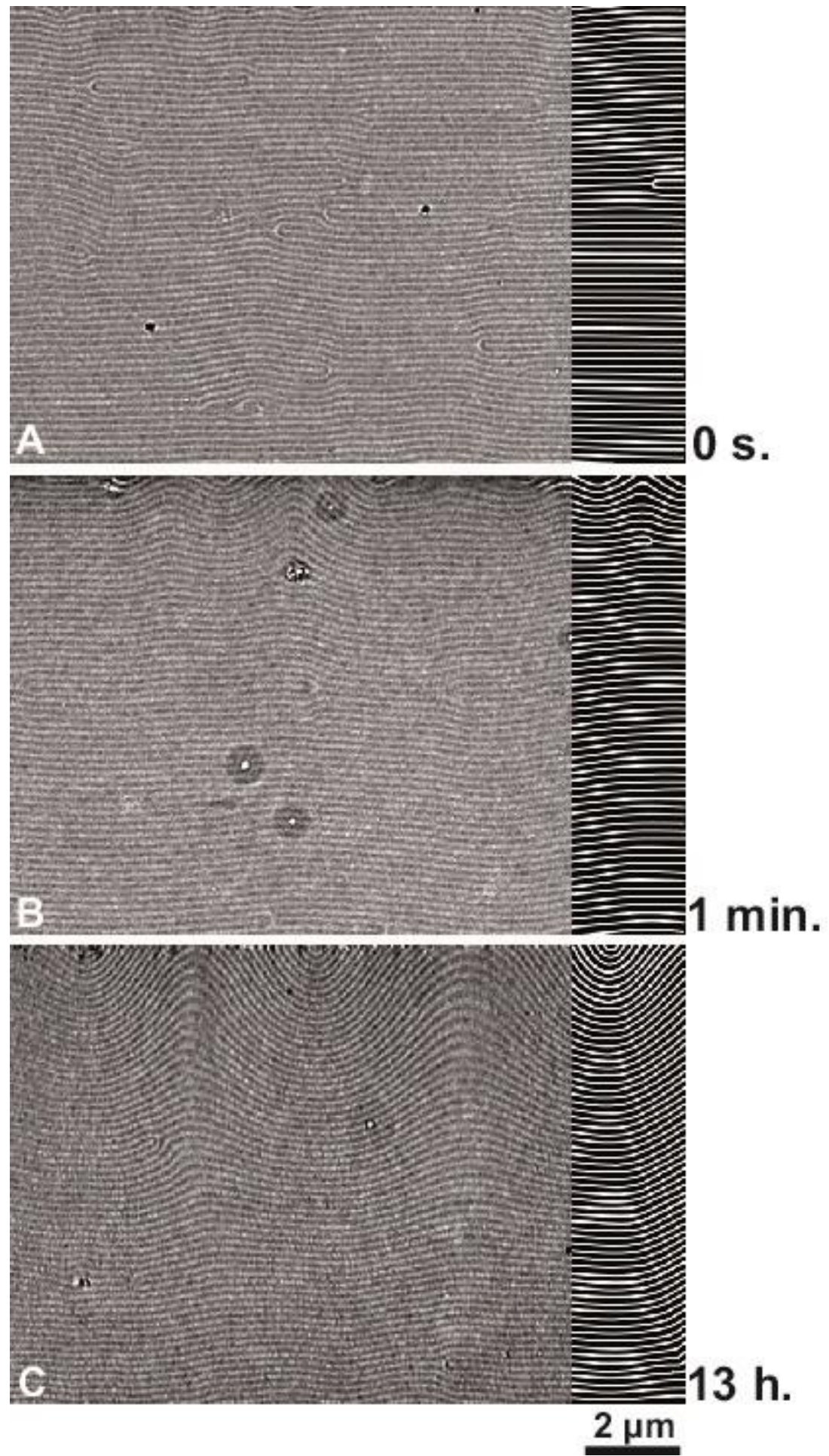

Fig. 2. Transmission electron microscopy (TEM) images of cross sections of cholesteric liquid crystalline films quenched at $140{ }^{\circ} \mathrm{C}$. The dark image on the right-hand side is the main image redrawn 
to facilitate observation of texture changes. (A) Without annealing: the fingerprint texture made of an array of periodic bright and dark lines is regularly parallel to both interfaces. The helix axis is everywhere perpendicular to the lines. The distance between two dark (or bright) lines represents the half pitch. (B) Annealing time = 1 minute: close to the air interface, the lines undulate, which indicates a change in the orientation of the helix axis. Further down, the lines remain parallel to the surfaces. (C) Annealing time $=13$ hours: the distortions have invaded the bulk. Only a very thin material thickness close to the substrate exhibits quasi parallel lines.

As soon as the annealing time reaches 1 minute, curved deformations appear close to the air interface (see fig. 2b, top) where anchoring of the molecules perpendicular to the interface is preferred (which is weaker than the planar anchoring on the substrate). At this time, the lines bend with respect to the free surface over a thickness less than $2 \mu \mathrm{m}$. As the annealing time increases up to 13 hours, large distortions progressively propagate into the bulk (fig. 2c) and an alternating series of concave and convex nested arcs develops. The time required for the propagation of distortions from the free surface into the bulk is related in part to the viscosity of the material at the given annealing temperature (if the temperature is increased above $140^{\circ} \mathrm{C}$, the annealing time required to obtain a targeted color is shorter, because the viscosity decreases when the temperature increases). Near the surface, the twisted structure cannot readily adapt to the anchoring conditions. One solution to this boundary problem is the creation of a series of alternating disclination lines at the surface. Nested-arc patterns develop because the anchoring at the free surface favors an oblique orientation of the helical axis relative to the surface. Such a situation, which has already been observed and discussed for large-pitch low-molar-mass CLCs [26, 27], corresponds to the director distribution of least elastic energy that allows for this surface anchoring together with the bulk planar structure.

AFM and SEM investigations show that the polygon cells are convex cones that exhibit double-spiral fingerprint patterns. From AFM imaging, we find that the height of the cones is 
in the $20-100 \mathrm{~nm}$ range. The equilibrium relief results from competition between surface energy and the bulk free energy. The energy is lowered by transforming the free surface into a cone, thus reducing the bulk distortion energy by the costs of surface energy. Discussing the formation of this series of valleys and crests at the free surface of LC films with antagonist anchoring is out of the scope of the present paper and this topic has already been examined for CLC [26, 27, 32] as well as nematic [33] LC films.

Figure 3 gives a three-dimensional sketch of the structure and shows the continuity between the cones exhibiting spiral patterns, the polygonal texture, and the nested arcs in the cross section. Although nested-arc cholesteric textures have already been observed by TEM in cross-sections of CLC oligomers [30, 34], the model used [34] does not apply to the nestedarc patterns in the fingerprint texture that we observe just below the free surface. Indeed, on one hand, this model is based on a unique object and, on the other hand, the experimental conditions correspond to cuts oblique to the helical axis, which explains why the authors describe a single focal conic domain that appears like a region of nested arcs embedded in a planar texture. Our experiments reveal an assembly of polygons and the required connections between polygons give priority to the arrangement we depict in fig. 3 . 


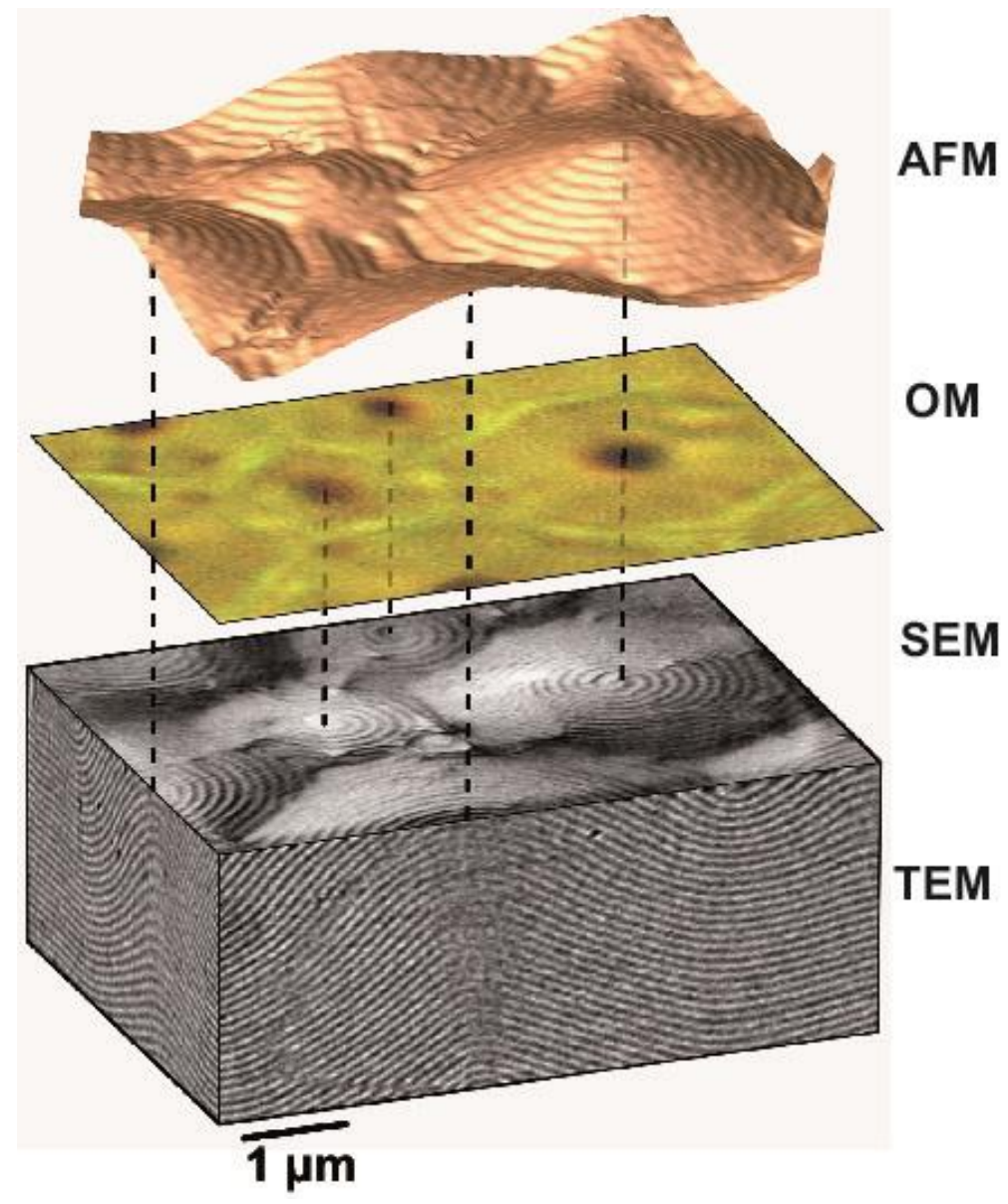

Fig. 3 Sketch summarizing the three-dimensional representation of cholesteric liquid crystalline films with polygonal texture as seen by optical microscopy (OM). AFM imaging reveals the cone shape of polygons, which are the locus of double-spiral patterned lines. The connection between oblique lines in TEM cross-sectional views and spiral patterns as seen in planar views by SEM is visible. Vertical dotted lines help visualize the coincidence between the central spots of cones from one image to the next.

\section{Description of the helical axis orientation}

The time-dependent band-shift scenario can be fully described with the help of a single parameter $\theta$, which is the angle between the normal to the film surface and the perpendicular to the lines at every point. Figure 4 a shows the $\theta$ distribution along the height $z$ for two extreme situations. Without annealing, the straight line focused at $\theta=0^{\circ}$ means that the helix 
axis is everywhere perpendicular to the surfaces (planar texture) whereas, after 18 hours, the much larger dispersion reveals strong deformations.
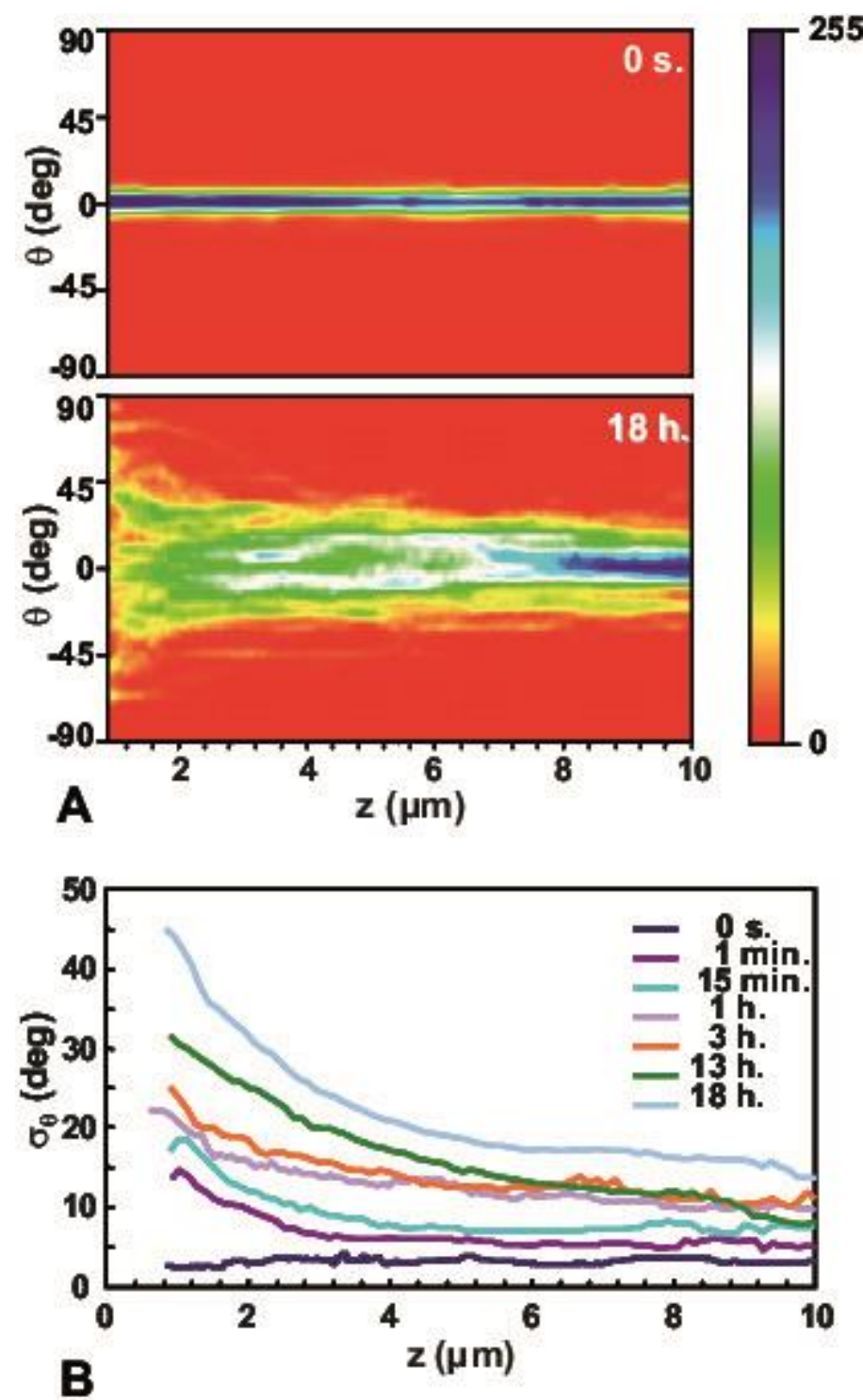

Fig. 4. Description of the helix axis orientation in the bulk of the material as a function of the annealing time. (A) Distribution of $\theta$ along the $z$ direction (height). Without annealing $(0 \mathrm{~h})$, the straight line at $\theta=0^{\circ}$ is the signature of a planar structure, whereas after $18 \mathrm{~h}$, the large dispersion leads to huge deformations that propagate through the entire volume. (B) Evolution of the $\theta$ meansquare profile $\sigma_{\theta}(z)$ for different annealing times. 
Near the free surface, the flat distribution in the range $\left[-90^{\circ} ;+90^{\circ}\right]$ reflects the existence of arced patterns and, in the bulk, the diagram shows the signature of residual undulations. A quantitative gauge of the helix deformation can be given using the $\theta$ mean-square parameter $\sigma_{\theta}$. The profile of $\sigma_{\theta}$ demonstrates the amplification of this distortion with annealing time (fig. $4 \mathrm{~b})$. The reflection color is shifted as a consequence of the unusual distribution of the helical axis orientation at the air-material interface; it is not related to modifications of molecular chirality or helical pitch (fig. S2 of ESI ${ }^{\dagger}$ ).

Because the cones exhibit a helical arrangement of molecules that rises in a double spiral pattern, we can indeed expect from this situation a quite complex polarization state for the reflected light. Here, the polarization-selectivity rule, which only applies when the incident direction is parallel to the helix axis, is not valid. Light propagating through the part of the material with nested arcs is reflected by a helical structure whose the orientation is continuously changing. By analogy, the description of the reflection properties in this region would originally correspond to the situation of oblique incidence [35], whose angle would change as the light propagates. In addition, two experimental facts explain why the transmittance level decreases with annealing time, both inside and outside the Bragg band (fig. 1b): First, the light striking the sample is reflected off axis and thus is not collected and, second, the boundaries of cone-like cells create defects that induce some light scattering.

\section{Numerical simulations of transmission spectra}

Numerical simulations indicate that the blue shift observed experimentally is related to distortions in the distribution of the helical axis. The simulations are based on information gained from TEM cross sections. A one-dimensional Bragg reflector is built with the $\theta$ profile along the thickness $z$. The dielectric material is mapped with ordinary (extraordinary) index $n_{\mathrm{o}}$ 
$=1.5\left(n_{\mathrm{e}}=1.7\right)$. The response of the system over a wide range of frequencies is obtained through the generation of a broadband Gaussian pulse. Simulated and experimental transmittance spectra are shown in fig. 5 for three typical annealing times: 5 minutes, 3 hours, and 18 hours. The correspondence between band-gap positions highlights the direct link between the deformations of the inner structure - accounted for by the $\theta$ dispersion-and the blue shift. We also note a good agreement for the bandwidths. The main difference between experimental and simulated curves is the consequence of the simplicity of the simulated set up. We can indeed specify that the high spatial coherence of the incident light, which is inherent to the one-dimensional configuration, induces interferences that result in overmodulation on both sides of the band gap. Moreover, this approach does not offer the opportunity to describe multiple reflections in the bulk, so no loss term is taken into account. Two-dimensional simulations are in progress to reproduce the actual complexity of the optical response of nested-arc structures.

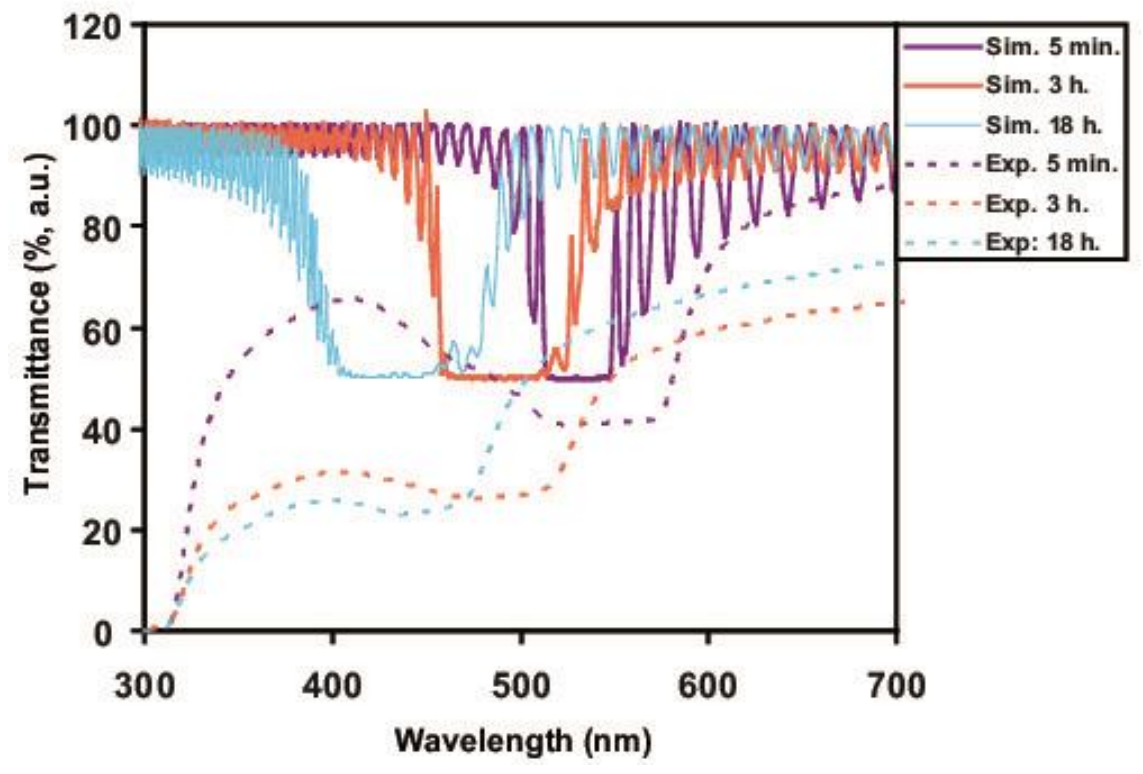

Fig. 5. Comparison between experimental (dashed lines) and simulated (solid lines) transmission spectra for different annealing times $(5 \mathrm{~min}, 3 \mathrm{~h}$, and $18 \mathrm{~h})$. 


\section{Analogy with the exoskeleton morphogenesis of some beetles}

Iridescent coloring of certain beetle cuticles results from helicoidal stacking of fibrous chitin layers [12]. Nested arc cholesteric textures have been observed by TEM in cross sections of cuticles of Chrysina (Plusiotis) Gloriosa [36, 37] and Manuka [38]. A closer analogy is provided by the (curved) cuticle structure of Chrysina Gloriosa beetle (see fig. 6) of whom our (flat) films might represent a synthetic version. This insect changes color because of the light-reflecting characteristics of the cone-like cells that make up its exoskeleton [37]. Below we suggest an analogy between the formation process of cone-like structures and the exoskeleton morphogenesis of Chrysina Gloriosa.
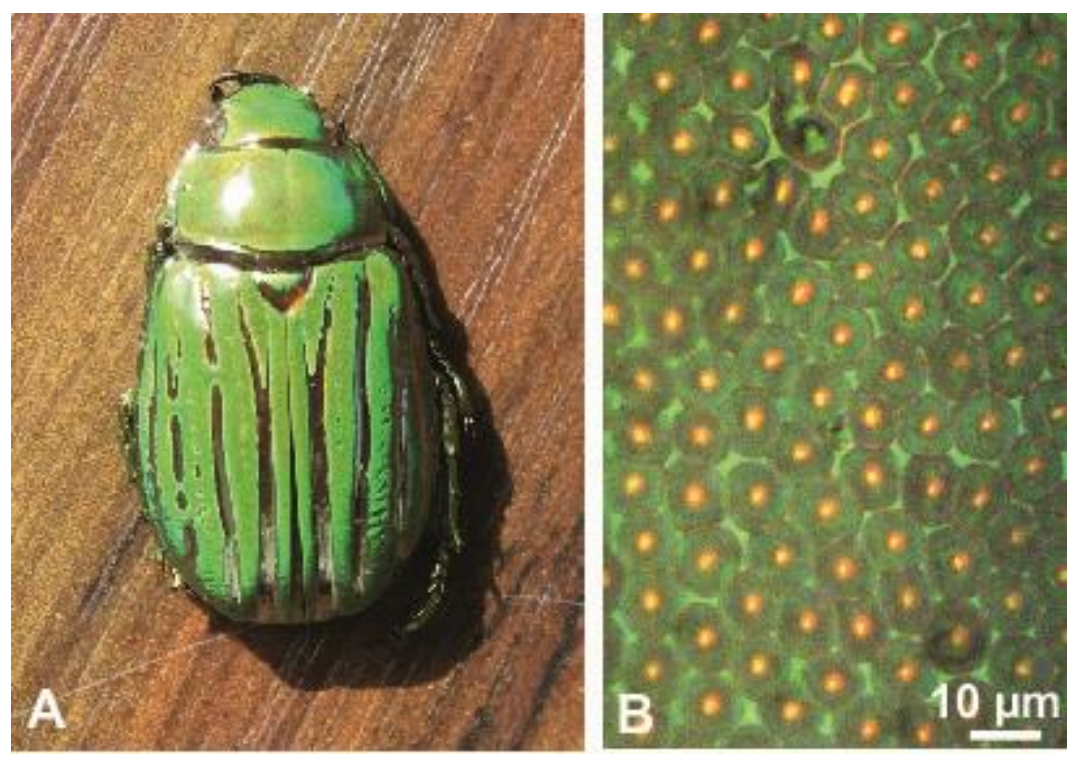

Fig. 6. Chrysina Gloriosa. (A) The beetle's exoskeleton includes green and silver stripes. (B) Optical micrograph of a green stripe exhibits a mosaic of dark greenish polygons with a central yellow spot and bright greenish reflections from the edges (reflection mode, unpolarized light).

Sclerotization is a complex process that confers stability and mechanical versatility to the insect exoskeleton [39, 40]. The structure of the exoskeleton of arthropods is determined 
genetically and by the formation conditions; for example, temperature and light intensity are important factors for deciding the filament patterns of chitin molecules [41]. The patterns of cuticle sclerotization are regulated by the underlying epidermis and other tissues through mechanisms that are poorly understood. The exoskeleton has a limited expandability for growth, thereby requiring insects to molt periodically to complete development to the adult stage. During each molting cycle, a new cuticle must be secreted by the epidermis and the old one partially digested to make escape possible. The understanding of the complex sclerotization process must consider the characteristics of the sclerozing agents, structural proteins, and chitin; their chemical interactions; the enzymes that direct the assembly; and stabilization of the exoskeleton outside the boundary of the epidermis. With regards to the latter factor, the formation process of the cone-like structures may provide insights into the exoskeleton morphogenesis of Chrysina Gloriosa. Deformations in the cholesteric organization at the air interface during the secretion of the cuticle by the epidermis, when the material is soft, may participate in part to the formation of the cellular texture (fig. 6b), before biopolymerization reactions (e.g., crosslinking between polypeptide chains) quickly stop the structure evolution and harden the exoskeleton.

\section{Conclusions}

The color selectivity of CLCs may be tuned by annealing an open film that has hybrid anchoring and a polygonal texture. The solid state of quenched films makes is possible to investigate the structure of film cross sections by TEM. We have shown that the blue shift of the reflection band is caused by interface-induced variations of the helical axis orientation, which progressively propagates into the volume with annealing time. The optical behavior is described using the angle between the surface normal and the helical axis. 
The optical behavior of polygonal cholesteric textures in relation with the evolution of geometrical features of nested-arc domains has not heretofore been reported, so limited knowledge was available on the optical and structural parameters to determine the optical response of the material. In this report, we provide a single control parameter (the annealing time) that, in return, offers the opportunity to selectively tune the reflection wavelength.

We fabricated chiral microreflectors by using a low-cost controllable thermal process and explain their optical properties using a geometric model based on their unique morphology. Because of the flexibility of this process, a wide range of designs may be implemented by using such a technique. The tuning of the transmission band only depends on the choice of the pristine cholesteric periodicity and the annealing time. This simplicity is important because the control of structures in soft materials forms the basis for applications such as displays, sensors, actuators, adaptive lenses, and beam-steering devices. The present elaboration procedure may provide a route for the fabrication of tunable micromirrors or microlens arrays: (i) the polygonal film may be seen as a self-assembly of spherical mirrors that present multiple reflections on the micron scale; (ii) the ESI includes a video clip that illustrates the microlens behavior of the cones. The rebuilt image along the propagation direction shows that light is focused towards the central spot by the curved surfaces.

Additionally, the conditions for polygonal texture formation provide insights to aid our understanding of the exoskeleton morphogenesis of beetles like Chrysina Gloriosa.

\section{Acknowledgements}

This work was supported by the Agence Nationale de la Recherche (ANR) under award number 07-NANO-032. We thank Dr. E. Hanelt from Wacker Chemie GmbH (Munchen, Germany) for providing us with oligomers and constant support; Mr. C. Bourgerette for his 
technical assistance with ultramicrotomy and TEM observations; and Drs. G. Seine and R. Péchou for AFM investigations.

\section{References and notes}

1 D. M. Small, Surface Chemistry of Biological Systems, 1970, ed. M. Blank, Plenum, NewYork, p. 55.

2 Y. Bouligand, M.-O. Soyer and S. Puiseux-Dao, Chromosoma, 1968, 24, 251-287.

3 F. Livolant and A. Leforestier, Prog. Polym. Sci., 1996, 21, 1115-1164.

4 A. D. Rey, Soft Matter, 2010, 6, 3402-3429.

5 D. G. Gray, Carbohyd. Polym., 1994, 25, 277-284.

6 I. W. Hamley, Soft Matter, 2010, 6, 1863-1871.

7 R. L. Trelstad, Developmental Biology, 1982, 92, 133-134.

8 M. M. Giraud-Guille, Calcif. Tissue Int., 1988, 42, 167-180.

9 M. M. Giraud, J. Castanet, F. J. Meunier, Y. Bouligand, Tissue and Cell, 1978, 10, 671-686.

10 Y. Bouligand, C. R. Acad. Sc. Paris Sér. D, 1965, 261, 3665-3668.

11 A. C. Neville and S. Caveney, Biol. Rev., 1969, 44, 531-562.

12 A. C. Neville, Biology of Fibrous Composites, 1993, Cambridge University Press, Cambridge.

13 Liquid Crystals, eds. S. J. Woltman, G. D Jay and G. P. Crawford, 2007, World Scientific, Singapore.

14 V. A. Belyakov and V. E. Dmitrienko, Sov. Sci. Rev. A Phys., 1989, 13, 1-212.

15 P.-G. de Gennes and J. Prost, The Physics of Liquid Crystals, 1993, Oxford University Press, Oxford, pp. 264-268.

16 M. Mitov, Les cristaux liquides, 2000, Presses Universitaires de France, Paris, pp. 44-49. 
17 The characteristics of the band of reflected light (position, width, intensity, polarization) may be externally controlled by temperature, mechanical pressure, electric or magnetic field, the angle or polarization of the incident light, electromagnetic radiation (photoinduced effects), or the sandwich-cell parameters (treatment of surfaces to induce a peculiar molecular anchoring or gap thickness).

18 T. J. Bunning and F.-H. Kreuzer, Trends in Polym. Sci., 1995, 3, 318-323.

19 F.-H. Kreuzer, N. Häberle, H. Leigeber, R. Maurer, J. Stohrer, and J. Weis, Cyclic liquid crystalline siloxanes, chemistry and applications. In Organosilicon Chemistry III, eds. N. Auner and J. Weiss, 1997, Wiley-VCH, Weinheim, pp. 566-586

20 C. Binet, M. Mitov and A. Boudet, Mol. Cryst. Liq. Cryst., 2000, 339, 111-123.

21 J. Pierron, A. Boudet, P. Sopena, M. Mitov and P. Sixou, Liq. Cryst., 1995, 19, 257-267.

22 A. Boudet, C. Binet, M. Mitov, C. Bourgerette and E. Boucher, Eur. Phys. J. E, 2000, 2, 247-253.

23 A. Boudet, M. Mitov, C. Bourgerette, T. Ondarçuhu and R. Coratger, Ultramicroscopy, 2001, 88, 219-229.

24 A. Taflove and S. C. Hagness, Computational Electrodynamics: The Finite-Difference Time-Domain Method, 2005, Artech House, Norwood, MA.

25 A. Farjadpour, D. Roundy, A. Rodriguez, M. Ibanescu, P. Bermel, J. D. Joannopoulos, S.G. Johnson and G. W. Burr, Optics Letters, 2006, 31, 2972-2974.

26 Y. Bouligand, J. Phys., 1972, 33, 715-736.

27 A. Saupe, Mol. Cryst. Liq. Cryst., 1973, 21, 211-238.

28 O. Lehmann, Flüssige Kristalle, 1904, Engelmann, Leipzig.

29 H. Hara, T. Satoh, T. Toya, S. Ikeda, S. Orii and J. Watanabe, Macromolecules 1988, 21, 14-19. 
30 T. J. Bunning, D. L. Vezie, P. F. Lloyd, P. D. Haaland, E. L. Thomas and W. W. Adams, Liq. Cryst., 1994, 16, 769-781.

31 J. Pierron, V. Tournier-Lasserve, P. Sopéna, A. Boudet, P . Sixou and M. Mitov, J. Phys. II France, 1995, 5, 1635-1647.

32 R. Meister, H. Dumoulin, M.-A. Hallé and P. Pieranski, J. Phys. II France, 1996, 6, 827844.

33 In Ref. 15, pp. 174-176.

34 R. Meister, M.-A. Hallé, H. Dumoulin and P. Pieranski, Phys. Rev E., 1996, 54, 37713782.

35 E. Miraldi, C. Oldano, P. I. Taverna and L. Trossi, Mol. Cryst. Liq. Cryst., 1983, 103, 155176.

36 A. Pace, Science, 1972, 176, 678-680.

37 V. Sharma, M. Crne, J. O. Park and M. Srinivasarao, Science, 2009, 325, 449-451.

38 L. De Silva, I. Hodgkinson, P. Murray, Q. H. Wu, M. Arnold, J. Leader, A. McNaughton, Electromagnetics, 2005, 25, 391-408.

39 S. O. Andersen, Ann. Rev. Entomol., 1979, 24, 29-61.

40 T. L. Hopkins and K. J. Kramer, Ann. Rev. Entomol., 1992, 37, 273-302.

41 A. C. Neville, and B. M. Luke, Tissue Cell, 1969, 1, 355-356. 
Electronic Supplementary Information (ESI) for Soft Matter

This journal is (C) The Royal Society of Chemistry 2011

\section{Supplementary Information}

\section{Color selectivity lent to a cholesteric liquid crystal by monitoring interface-induced deformations}

Gonzague Agez, Rajaa Bitar and Michel Mitov

Centre d'Elaboration de Matériaux et d'Etudes Structurales (CEMES), CNRS, Univ. Toulouse, 29 rue J. Marvig, 31055 Toulouse cedex 4, France

\section{Characteristics of Bragg band}

At normal incidence, the bandwidth is $\Delta \lambda=p \Delta n$, where $\Delta n=n_{\mathrm{e}}-n_{\mathrm{o}}$ is the birefringence ( $n_{\mathrm{e}}$ and $n_{0}$ are the extraordinary and ordinary refractive indices, respectively). The bandwidth is measured at half maximum. Figure S1 shows the evolution of the mean position and width of the Bragg band as a function of annealing time. We deduce that the evolution of the optical properties mainly corresponds to a band shift toward shorter wavelengths without a significant broadening of the bandwidth.

\section{Periodicity measurements in TEM micrographs}

The fingerprint periodicity is the distance between two lines with same contrast and represents the half pitch. Figure S2 shows the variation of the periodicity as a function of the $z$ position as a function of annealing time. The periodicity is everywhere measured in a direction perpendicular to the lines using numerical two-dimensional Fourier transform from TEM cross-sectional images. The periodicity is quite constant throughout the volume. We deduce that an eventual pitch variation does not apply to the optical behavior. 


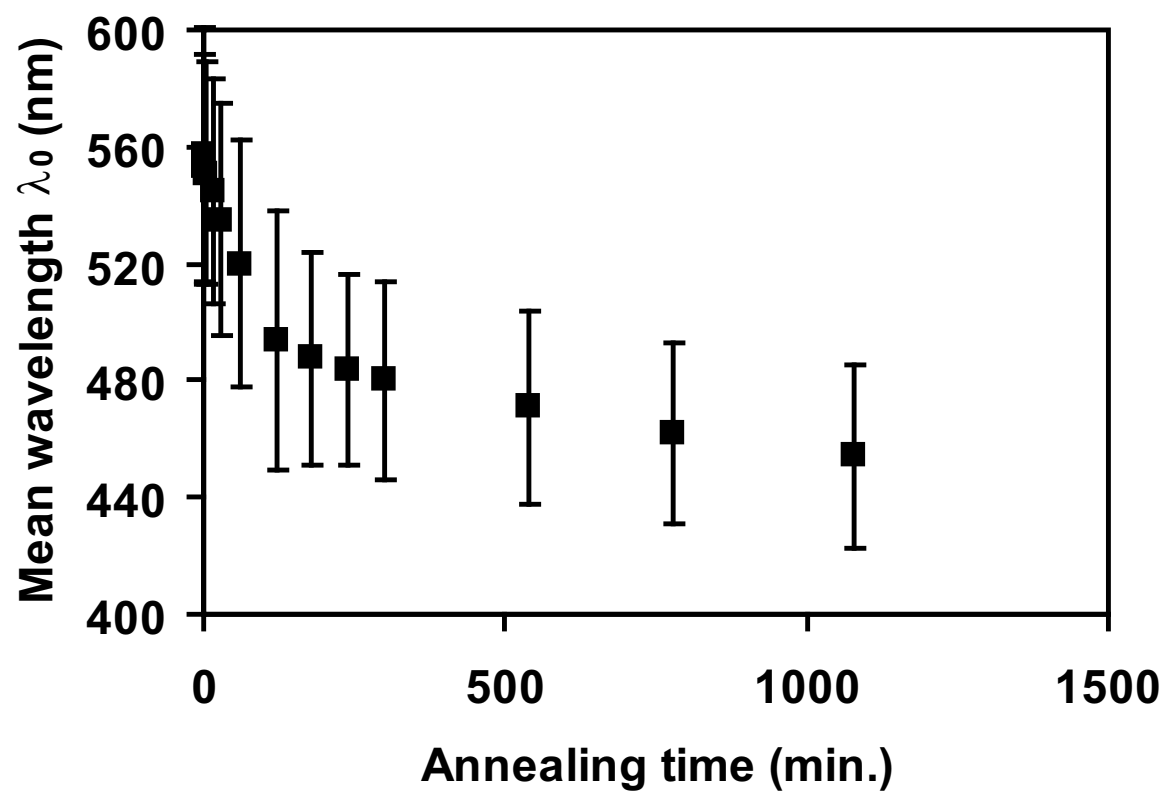

Fig. S1. Position and width (bars) of the band gap as a function of the annealing time.

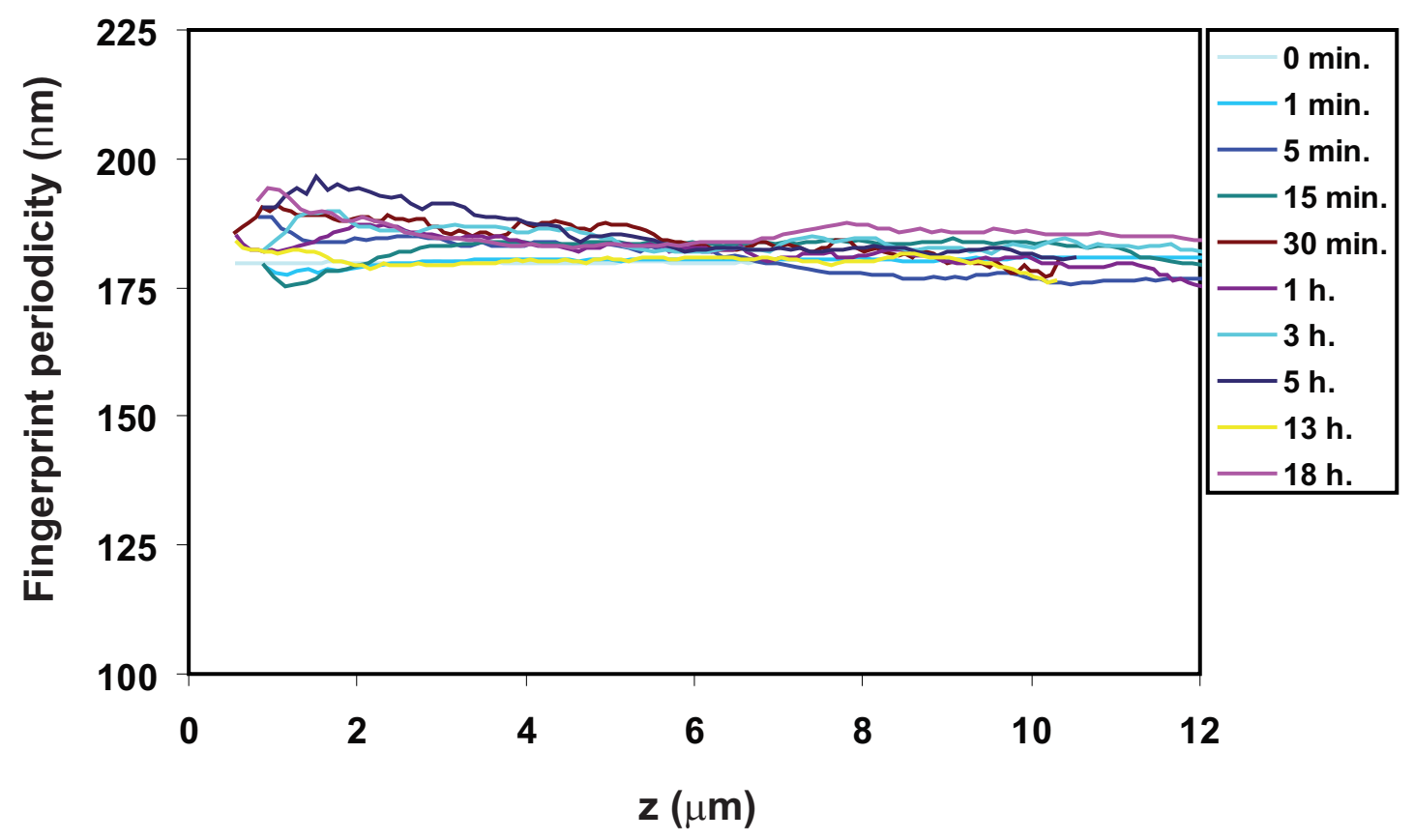

Fig. S2. Fingerprint periodicity as a function of the height $z$ within the film and for different annealing times. 
Supplementary Movie Legend: Movie illustrating the microlens effect (transmission mode, annealing time $=9 \mathrm{~h}$ ). (A, upper part) The movie is built from a stack of optical micrographs of polygonal texture. Each image has been recorded with the microscope focus varying from below to above the free surface (the counter indicates the depth of focus $z$ ). (B, lower part) Orthogonal view of the stack extracted from the dashed line as shown in A. The dynamic yellow line gives the correspondence between the depth of focus $z$ and the current $x-y$ slice displayed in A. It emphasizes that the focus point is located very close to the free surface $(z=$ 0). (QuickTime; 1.18 MB). 